\section{Response latency and serial position in short-term memory*}

\author{
ALFRED H. FUCHS \\ Bowdoin College, Brunswick, Maine 04011
}

A serial position effect was observed in latency scores when a visually presented test element was correctly identified as having a particular serial location in a memorized serial list. The effect was not observed in a choice reaction-time task which made similar response requirements. The data suggest that the serial position effect for latency scores, as for error scores, arise in memory tasks in which the identification of a position in a list is critical for correct responding.

A self-terminating search has been postulated to account for latency data obtained in a memory task in which a digit from a sequentially presented list of digits constituted the recall cue (probe) for the immediately subsequent digit (Sternberg, 1967). Recall latencies, on the average, were fastest for Serial Position 2 (tested by the first item in series) and increased linearly, with the longest response latency associated with the last position in the sequence; a search process, rather than direct access to items, was postulated since average latencies increased as a function of list length. The search task required locating the probe before a response could be made; scanning to locate could begin with the first item in the list and proceed in a forward order, examining each item in turn until the test and subsequent response items were identified and the search terminated, or, the search might begin with any item randomly in a "circular" search strategy. Both strategies were reflected in the data, with some Ss showing linear latency increases over serial position (search began with first word), while others showed flat latency functions over serial position (random starting point).

Evidence for a recency effect was obtained in a different recall task (Fuchs, 1969). Lists of five words were presented, one in each of five spatial locations, and the cue for recall was an asterisk which appeared in a serial location formerly occupied by a word. Errors were distributed in a

\footnotetext{
*This research was conducted while the author served as Professor of Psychology at Victoria University of Wellington, New Zcaland. Funds for the apparatus employed were provided by a grant from the New Zealand University Grants Committee to $L$. B. Brown and J. Dutch. The writer is grateful to them for making the apparatus available, and to Mr. Fred Lane for the construction of timers and circuits which made the separate pieces a work able system. Funds for reprints were provided by a grant from the Bowdoin College Faculty Research Fund. James L. Fozard read earlier drafts of this paper and provided helpful suggestions and comments.
}

bowed serial position curve. The latency data, obtained under instructions which emphasized rapid and accurate responding, are reported in Table 1 for one condition of that study (two presentations, 4-sec retention interval). The latencies show a serial position effect, with the inflection of the bowed curve at Position 3. The latencies over the middle positions show somewhat greater variablility than the first and last positions; if the median is used as the average, the inflection of the curve is at Serial Position 4. The number of responses at each position were, for Positions 1-5, respectively, $39 / 45$, $28 / 45,21 / 45,21 / 45$, and $41 / 45$. In view of the few responses at Positions 3 and 4 , it is perhaps not surprising that the medians should be somewhat discrepant from the means.

Although there were a number of differences between the Sternberg (1967) and Fuchs (1969) experiments, both required the recall of one item from a memorized string of items. One difference was the nature of the search task as defined by the cue employed. The use of an item probe may result in an item-by-item sequential search to locate the probe in the list, ". . . followed by a shift from test item to adjacent response item [Sternberg, 1967, p. 55]." This cue, a "sequential probe [Murdock, 1968]" may well emphasize the items within a sequence, rather than their serial order or location; the search is directed toward "the item which comes after ." The position of items in the sequence was obscured in the Sternberg (1967) data because the items were presented successively in the same spatial location. Furthermore, the sequential probe emphasized relations between words as the basis for recall, and position information, even if available for items, could be ignored without interfering with correct recall. The asterisk, or positional cue, on the other hand, may direct search toward "the item which appears in position ," and, therefore, emphasizes the relation of an item to a serial location.

A further difference in the tasks is the high error rate reported for the Fuchs (1969) data in Table 1 (57\%) compared with the overall error rate in the Sternberg (1967) data (8.2\%). The failure of average latency to change over set size with increased frequency of stimulus presentation in a sequential probe task has been interpreted to indicate that latency measures an invariant retrieval process, while errors represent failures of learning and retention (Sternberg, 1969). If so, the observed serial position effect of latency under high error conditions may also reflect learning or retention faults and not retrieval processes.

In order to examine further the bowed serial position curve for latency as a retrieval process, a task was investigated in which an error rate, more comparable to the Sternberg (1967) results, could be achieved. The task required identifying the serial position of a test item in a memorized list of items. Thus, position information was critical to the task, and learning, as reflected in error rate, was complete for all items. If the bowed serial position curve for latency appears under low error rate conditions, the interpretation of latency as an indication of the retrieval process is reinforced, although the nature of the search process needs reexamination.

\section{SUBJECTS}

Four male and four female volunteers from the second year psychology course at Victoria University of Wellington, New Zealand, served as Ss.

\section{APPARATUS AND PROCEDURE}

The apparatus employed consisted of a response panel on which a set of five white $1 / 2$-in. pushbuttons were arranged in a semicircle; each button was $3 \mathrm{in}$. from a red $1 / 2$-in. center button. A choice reaction-time task (CRT) was used to familiarize Ss with the response required in the STM task. Five indicator lights were arranged in identical fashion to the pushbuttons, in order to maximize S-R compatibility (Fitts \& Seegar, 1953); Ss rested the index finger of the right hand on the red pushbutton and were required to press the white button which corresponded to whichever light was turned on. Each $\mathrm{S}$ received 45 trials; each of the five serial positions was tested nine times. The order of testing positions was random within blocks of 15 trials.

Immediately following the CRT training, the STM task was introduced. Slides containing strings of five four-letter words (Fuchs, 1969) were projected on a screen; the words were 
Table 1

Mean and Standard Deviation Latency (in Sec) of Responding to Each Serial Position in a Recall Task (Fuchs, 1969) and the CRT and STM Tasks of the Present Experiment

\begin{tabular}{llccccc}
\hline \multirow{2}{*}{ Fuchs, 1969 } & Mean & 2.279 & 2.442 & 2.950 & 2.730 & 2.15 \\
& SD & .870 & 1.20 & 1.23 & 1.21 & .937 \\
CRT & Mean & .624 & .629 & .600 & .613 & .599 \\
& SD & .173 & .132 & .085 & .113 & .113 \\
STM & Mean & .949 & 1.01 & 1.32 & 1.24 & .932 \\
\hline
\end{tabular}

arranged in the same semicircular patterns as the pushbuttons. Each string received two presentations, at each of which $S$ read the words aloud. A 3 -sec retention interval was filled with a blank dark screen; light from the test slide activated a photocell and, in turn, a timer, which was stopped when $S$ responded. No task was assigned to $\mathbf{S}$ during this retention interval, during which $\mathrm{E}$ recorded latency, reset the timer, and pressed a key to initiate the test slide after 3 sec had elapsed. Retention interval duration was recorded on each trial to measure E's performance, since the timing of the retention interval was controlled manually by $\mathrm{E}$ with the aid of a stopwatch. 1

A test slide contained one of the five words from the string in a position corresponding to the red pushbutton on the response panel. All Ss were instructed to press the white button corresponding to the position the word had occupied in the string. Each serial position was tested five times in a sequence arranged so that each position followed every other position, including itself, equally often. Ten practice trials preceded the data collection trials to familarize Ss with the task. Instructions for both tasks emphasized speed consistent with accuracy; Ss were asked to try to make no errors.

\section{RESULTS}

In the STM task, nine errors were committed in 200 trials ( 8 Ss by 25 trials) for an error rate of $4.5 \%$. This compares favorably with an error rate of $8.2 \%$ over list lengths up to seven items (Sternberg, 1967), but is probably slightly higher than that indicated for the five-item lists alone.

Table 1 contains the means and standard deviations of the correct response latencies for each serial position in the STM task, in which S was to indicate the position of a word in the list, and the corresponding data for the CRT task, in which each $S$ had only to respond to the onset of a light. Recall latencies in the STM task are characterized by a bowed serial position curve; the longest latency is associated with Serial Position 3 , as in the Fuchs (1969) data. In contrast, the lack of a serial position effect in the choice reaction-time task indicates that the latencies in STM are not a function of the response requirements alone. These effects can be attributed to the differential time required to recall items at different serial positions from memory.

In order to evaluate statistically the effects apparent in Table 1 , a mean reaction time was computed for each $S$ in the CRT and STM tasks. A difference score was determined by subtracting the average CRT latency from the average STM latency for each $\mathrm{S}$. The difference scores showed significant effects for serial position $[F(4,28)=10.89, p<.001]$.

\section{DISCUSSION}

In the present study, the serial position of a test item was the information to be recalled. Since each series of words was presented twice, the order and position of words in the strings should have been reasonably well-learned (Fuchs, 1969); the low number of errors shows that this was the case. A learning interpretation of the data is not ruled out by the low error rate since latency is traditionally employed as a measure of learning. Degree of learning may well vary over serial position and contribute to the results obtained in the present study. Such an interpretation could assume direct access to each item, as in a search model in which the search can begin at any position. Given position information in the test item, that position is searched directly, and latencies reflect relative strengths of position-item associations. This interpretation could be tested by examining the effects of increased presentation upon latency at each serial position. For the present, it can only be noted that the interpretation available in the probe, necessary for recall, and utilized in responding. Under these conditions, direct access and search have been postulated to account for bowed serial position effects for latency scores (Moss \& Sharac, 1970).

A sequential self-terminating search process can account for the data if search is possible in both forward and backward sequential orders, with starting points at either the first or last serial position. If the test item provides some information about its location in the list, the search is requires that positon information be initiated at the end of the list closest to that location. For the third position, search might begin at either end, and in either case, Serial Position 3 responses would have the longest latencies. This modification of Sternberg's (1967) self-terminating search model would leave intact the fundamental interpretation of latency as the time required to carry out a sequential search and arrive at a serial location and provide for the possibility that information about serial position, available in the probe (an "order tag;" Yntema \& Trask, 1963), can be used if it facilitates correct recall. Search strategies with a consistently random starting point, or always beginning with the first item, fail to utilize position information, and these strategies may reflect the lack of such information or its lack of relevance to the Sternberg (1967) task in which position is unimportant in determining the response.

\section{REFERENCES}

FITTS, P. M \& SEEGAR, C. M. S-R compatability: Spatial characteristics of stimulus and response codes. Journal of Experimental Psychology, 1953, 46, 199-210.

FUCHS, A. H, Recall for order and content of serial word lists in short-term memory. Journal of Experimental Psychology, $1969,82,14-21$.

MOSS, S. M., \& SHARAC, J. Accuracy and latency in short-term memory: Evidence for a dual retrieval process. Journal of Experimental Psychology, $1970,84$. $40-46$.

MURDOCK, B. B., JR. Serial order effects in short-term memory. Journal of Experimental Psychology, 1968, 76, (4. Pt. 2) 1-15.

STERNBERG, S. Retrieval of contextual information from memory. Psychonomic Science, $1967,8,55-56$.

STERNBERG, S. Memory scanning: Mental processes revealed by reaction time experiments. American Scientist, 1969 $57,421-457$

Y NTEMA, D. B., \& TRASK, F. P. Recall as a search process. Journal of Verbal Learning \& Verbal Behavior, 1963, 2. 65-74.

\section{NOTE}

1. Because $E$ controlled the retention interval duration manually, knowledge of the serial position being tested could have affected the duration of the retention interval on any trial, and, indirectly, the obtained latency. To assess this possible bias, the recorded duration of retention intervals was analyzed; by chance, three of the first four $S s$ to participate in the experiment were females, and the mean retention intervals were 3.40 sec for females and 3.23 sec for males. This difference was significant $[F(1,30)=8.44, p<.01]$, but no significant effects were obtained for serial position or the Sex by Serial Position interaction. Thus, latency differences across serial position are not a function of $E$ bias in determining retention intervals. Further, the significantly longer retention interval for females did not produce significantly longer response latencies, since the only significant effect in the analysis of STM latencies was obtained for serial position $\lceil F(4,24)=12.88, p<.001\}$. 